\title{
Title:
}

\section{Capsule enteroscopy versus scintigraphy for the diagnosis of obscure gastrointestinal bleeding}

\section{Authors:}

Miguel Mascarenhas Saraiva, Tiago Ribeiro, Patrícia Andrade, Hélder Cardoso, Guilherme Macedo

DOI: $10.17235 /$ reed.2021.7633/2020

Link: PubMed (Epub ahead of print)

Please cite this article as:

Mascarenhas Saraiva Miguel, Ribeiro Tiago, Andrade Patrícia, Cardoso Hélder, Macedo Guilherme. Capsule enteroscopy versus scintigraphy for the diagnosis of obscure gastrointestinal bleeding. Rev Esp Enferm Dig 2021. doi: 10.17235/reed.2021.7633/2020.

This is a PDF file of an unedited manuscript that has been accepted for publication. As a service to our customers we are providing this early version of the manuscript. The manuscript will undergo copyediting, typesetting, and review of the resulting proof before it is published in its final form. Please note that during the production process errors may be discovered which could affect the content, and all legal disclaimers that apply to the journal pertain. 
OR 7633

Capsule enteroscopy versus radionuclide scintigraphy for the diagnosis of obscure gastrointestinal bleeding

Miguel Mascarenhas Saraiva ${ }^{1,2}$, Tiago Ribeiro ${ }^{1,2}$, Patrícia Andrade ${ }^{1,2,3}$, Hélder Cardoso $^{1,2,3}$, Guilherme Macedo $0^{1,2,3}$

${ }^{1}$ Department of Gastroenterology. Centro Hospitalar Universitário de São João. ²WGO Gastroenterology and Hepatology Training Center. ${ }^{3}$ Faculdade de Medicina. Universidade do Porto. Porto, Portugal

Received: $11 / 11 / 2020$

Accepted: $10 / 05 / 2021$

Correspondence: Miguel José da Quinta e Costa de Mascarenhas Saraiva. Department of Gastroenterology. Centro Hospitalar Universitário de São João. Alameda Prof. Hernâni Monteiro. 4202-451 Porto, Portugal e-mail: miguelmascarenhassaraiva@gmail.com

Authors contributions: Miguel Mascarenhas Saraiva: data collection and analysis, drafting and revision of the manuscript. Tiago Ribeiro: data collection and analysis, drafting and revision of the manuscript. Patrícia Andrade: critical revision of the manuscript. Hélder Cardoso: study design, data analysis and critical revision of the manuscript. Guilherme Macedo: critical revision and final approval of the manuscript. Conflicts of interest: nothing to disclose.

\section{ABSTRACT}

Background: capsule enteroscopy (CE) and ${ }^{99 \mathrm{~m}} \mathrm{Tc}$ red blood cell (RBC) scintigraphy are frequently used in the investigation of obscure gastrointestinal bleeding (OGIB). There are few data comparing both diagnostic modalities. This study aimed to assess the 
performance of CE and scintigraphy for the diagnosis of OGIB.

Methods: patients who underwent CE and scintigraphy for OGIB were selected and analyzed retrospectively. The hemorrhagic potential of CE findings was rated using Saurin's classification. The concordance between both diagnostic techniques for bleeding detection and localization was analyzed.

Results: eighty-five patients (62\% female), with a median age of 63 years, were included in the study. Capsule enteroscopy identified 37 patients (43\%) with high hemorrhagic potential (P2) lesions. Most scintigraphy exams were positive for gastrointestinal bleeding ( $82 \%)$. No concordance was found in the detection of lesions with hemorrhagic potential between CE and scintigraphy (kappa <0). The distribution of $\mathrm{P} 0, \mathrm{P} 1$ and $\mathrm{P} 2$ findings was similar in patients with positive or negative scintigraphy $(p=0.526)$. There was no agreement regarding the location of $P 2$ findings in CE and the bleeding detected by scintigraphy (kappa < 0). Patients with P2 lesions had significantly lower median levels of hemoglobin $(p=0.002)$ at presentation. No significant differences were found in hemoglobin values between patients with positive and negative scintigraphy $(p=0.058)$.

Conclusion: significant diagnostic discrepancy was observed between CE and scintigraphy. The findings of CE correlated better with hemoglobin values at presentation than scintigraphy results. Therefore, scintigraphy does not appear to be useful in the diagnostic workup of OGIB.

Keywords: Capsule endoscopy. Scintigraphy. Gastrointestinal hemorrhage. Irondeficiency anemia.

\section{INTRODUCTION}

Obscure gastrointestinal bleeding comprises cases of gastrointestinal bleeding, either overt or occult, with unremarkable conventional endoscopic study (upper endoscopy and colonoscopy). This entity accounts for $5 \%$ of all cases of gastrointestinal hemorrhage. The majority of these cases are due to small-bowel bleeding, which poses 
both diagnostic and therapeutic challenges (1).

The advent of enteroscopy techniques that allow a complete bowel inspection, most notably capsule endoscopy (CE), has revolutionized the approach to these patients. In fact, it is recommended by most clinical practice guidelines as the first line in the study of occult gastrointestinal bleeding (OGIB) (1). Evidence suggests that CE is superior to push-enteroscopy, radiographic small-bowel studies, and angiography (2-4). Furthermore, CE has performed similar to double-balloon enteroscopy (DBE) and may further increase the yield of DBE $(5,6)$.

${ }^{99 m}$ Tc RBC scintigraphy is a widely used and highly sensitive method for the detection of gastrointestinal bleeding. It identifies bleeding with rates as low as $0.04 \mathrm{~mL} / \mathrm{min}$ (7). However, it has been demonstrated that it correlates poorly with other diagnostic tests, such as angiography, and does not predict further diagnostic and therapeutic course $(8,9)$.

To date, there are no studies correlating the findings of ${ }^{99 m} \mathrm{Tc}$ RBC scintigraphy and CE. Therefore, this study aimed to assess the diagnostic performance of ${ }^{99 \mathrm{~m} T C} \mathrm{RBC}$ scintigraphy and CE in patients with OGIB, either overt or occult.

\section{PATIENTS AND METHODS}

\section{Participants}

A total of 462 consecutive adult patients who underwent CE for OGIB between January 2016 and December 2019, at the Department of Gastroenterology, Centro Hospitalar Universitário de São João, in Portugal were included. All patients had a previous negative upper endoscopy and colonoscopy for potential sources of bleeding. Nondigestive sources of bleeding were disclosed. Clinical data from 85 patients who also underwent ${ }^{99 m}$ Tc RBC scintigraphy were retrospectively analyzed. Concordance between both diagnostic techniques regarding bleeding detection and location was evaluated. Data regarding gender, age, indication for examination, and hemoglobin levels was obtained. The study was approved by the ethics committee of Centro Hospitalar Universitário de São João. 


\section{Capsule enteroscopy}

Patients with OGIB underwent CE using the PillCam SB3 ${ }^{\circ}$ system (Medtronic, Minneapolis, Minnesota, USA). Bowel preparation before CE, guidance for the initiation of oral diet, and completion of the examination followed previously published guidelines (10). The hemorrhagic potential of endoscopic findings was determined through the application of Saurin's classification - PO: no hemorrhagic potential including visible submucosal veins, diverticula without blood, and nodules; P1: uncertain hemorrhagic potential, including red spots or small erosions; P2: high hemorrhagic potential such as angiomata, large ulcerations, tumors, and varices (11). CE was considered positive if P2 findings or blood pooling were reported.

\section{Scintigraphy}

The ${ }^{99 m}$ Tc RBC scintigraphy was performed by in-vivo radiolabeling of autologous RBCs with ${ }^{99 m}$ Technetium-pertechnetate. Dynamic images were obtained from the abdominal region. Image acquisition was performed for up to $24 \mathrm{~h}$ in some cases. The images were reviewed by nuclear medicine specialists. Suspected bleeding was reported if there was evidence of luminal extravasation of labeling material. Results available for review were compared with $\mathrm{CE}$ results to assess the concordance between both diagnostic systems.

\section{Statistical analysis}

Baseline characteristics of the patients, including age, gender, hemoglobin value, and time between CE and scintigraphy were registered. Continuous variables are described as medians and respective interquartile range (IQR, $25^{\text {th }}$ to $75^{\text {th }}$ percentile). Categorical variables are expressed as frequencies $(n)$ and percentages. Continuous variables were compared using the Mann-Whitney or Kruskal-Wallis tests, as appropriate. Categorical variables were analyzed using Fisher's exact test. The degree of agreement between VCE and scintigraphy findings was measured by the kappa $(\kappa)$ statistic. Correlations were assessed using Spearman's rank-order coefficient. A $p<0.05$ was considered statistically significant. Data analysis was performed using the Statistical Package for Social Sciences version 26 (SPSS ${ }^{\oplus}$, IBM Corp., Armonk, New York, USA). 


\section{RESULTS}

Eighty-five patients underwent both CE and ${ }^{99 \mathrm{~m}} \mathrm{Tc}$ RBC scintigraphy for the investigation of OGIB, and were included in the study for analysis. In most patients (86\%), these examinations were requested due to unexplained iron-deficient anemia (occult OGIB). Sixty-two percent of the patients were female, with a median age of 63 years (IQR, 51-74). Scintigraphy was performed before CE in $91 \%$ of the patients. The median time between the two tests was 4 months (IQR, 1.5-7.0). ${ }^{99 m} \mathrm{Tc}$ RBC scintigraphy identified bleeding in $82 \%$ of patients. The overall diagnostic yield of CE was $43 \%$. The most commonly described lesion was angiectasia (37\%), followed by erosions (20\%) and ulcers (12\%). Descriptive statistics are summarized in table 1.

\section{Concordance between CE and scintigraphy findings}

The distribution of CE findings according to scintigraphy results is shown in table 2 . The distribution of lesions with different bleeding potential (P0, P1 or P2) did not differ between those with positive (P0: $46 \%$; P1: $11 \%$; P2: $43 \%$ ) or negative (P0: 33 \%; P1: $20 \%$; P2: $47 \%$ ) scintigraphy $(p=0.526)$. There was no concordance between the results of scintigraphy and the identification of lesions with hemorrhagic potential $(\kappa=$ -0.020). Similarly, there was no concordance between the topography of the bleeding in the scintigraphy and the location of a potential bleeding lesion in CE ( $\kappa=-0.031)$.

As the interval between scintigraphy was highly variable, a sub-analysis was performed in order to assess the influence of this interval in the obtained results (Table 3 ). In patients where CE and scintigraphy were performed with an interval larger than 2 months, the distribution of P0, P1 and P2 lesions did not differ between those with positive (P0: $50 \%$; P1: $10 \%$; P2: $40 \%$ ) or negative (P0: $40 \%$; P1: $20 \%$; P2: $40 \%$ ) scintigraphy $(p=0.670)$. Similar results were observed for those when the interval between CE and scintigraphy did not exceed 2 months ( $p=0.772$ ) (Table 3 ).

\section{Relationship between the presence of hemorrhage and hemoglobin values}

As shown in table 4, no statistically significant difference was found in hemoglobin levels at presentation between patients with positive or negative ${ }^{99 m} T$ C RBC 
scintigraphy $(p=0.058)$. In contrast, those with lesions with a high bleeding potential in CE had significantly lower hemoglobin values $(p=0.002)$. A post-hoc analysis identified a significant difference between those with P2 lesions and patients with lesions without bleeding potential (Fig. 1).

The presence of lesions with high bleeding potential was significantly correlated to lower hemoglobin levels (Spearman's $\rho=-0.381, p<0.01$ ), whereas this correlation was not apparent for those with positive scintigraphy $(p=0.058)$.

\section{DISCUSSION}

Scintigraphy with radiolabeled RBCs has been used for the study of gastrointestinal bleeding for decades. In fact, it is reported as extremely sensitive, detecting bleeding with rates as low as $0.04 \mathrm{~mL} /$ minute (7). Furthermore, it does not require bowel preparation and, due to its prolonged acquisition time, allows for the detection of intermittent bleeding. However, subjective interpretation of nuclear scans is possible, which may contribute to inaccuracy in the location of the bleeding site (7). Therefore, several studies have demonstrated the limited utility of ${ }^{99 \mathrm{~m}} \mathrm{Tc}$ RBC scintigraphy in directing further diagnostic and therapeutic approaches $(8,9,12)$. Over the last few years, several studies have evaluated the correlation between scintigraphic findings and the results of other imaging tests, most frequently catheter angiography or computerized tomography (CT) angiography. A positive nuclear scan is a poor predictor of positive angiography (13). The correlation between these tests (positive ${ }^{99 \mathrm{~m}} \mathrm{Tc}$ RBC scintigraphy and positive angiography) has been estimated to occur in $21-54 \%$ of cases $(7-9,12,14)$. This may be explained by the increased sensitivity of scintigraphy and lowrate of intermittent bleeding detected by nuclear scans but not angiography. It has been demonstrated that positive scintigraphy does not predict subsequent diagnostic or therapeutic steps, and its real impact on patient management is dubious (9). Therefore, its current role in the investigation of OGIB is questionable.

Capsule enteroscopy has revolutionized the approach to patients with OGIB, either overt or occult. OGIB is the most frequent indication for CE (15). CE has a positive safety profile and allows a minimally invasive evaluation of the full length of the small bowel in a cost-effective manner (16). Similar to scintigraphy, it allows for the 
detection of intermittent bleeding due to its prolonged video acquisition time. Thus, CE is the first-line approach for further evaluation of patients with OGIB, after previous normal conventional endoscopy $(1,17)$. Capsule retention, the most feared complication, occurs in $1.4 \%$ of patients (15). Adding to its exceptional tolerability and safety, CE has been shown to be an accurate diagnostic method, with a high sensitivity (95\%) and specificity (75\%). A meta-analysis has estimated an overall diagnostic yield of CE for OGIB of almost $62 \%$ (5). Furthermore, these values were enhanced if CE was performed early after bleeding onset or in those with overt OGIB (18-20). Factors associated with an increased probability of positive CE include the use of nonsteroidal anti-inflammatory drugs, diagnosis of connective tissue disease, ongoing overt bleeding, and lower hemoglobin values with increased transfusion needs (20-22). Moreover, the usefulness of CE was established, providing higher diagnostic yields and reducing costs in high-burden investigations of OGIB patients (16). In fact, most patients with positive CE undergo a subsequent etiologic investigation, which leads to specific therapy in up to $80 \%$ of cases (19-21). Studies comparing the diagnostic yields of different examinations for the evaluation of the small bowel, either radiologic or endoscopic, indicated an overall superiority of CE. CE outperformed angiography $(53 \%$ vs. $20 \%$ ), CT angiography ( $72 \%$ vs. $56 \%$ ), and push enteroscopy (63\% vs. $28 \%$ ) $(2,4,23)$. The diagnostic yield of CE was shown to be similar to that of the much more invasive DBE (5). Application of CE before DBE increased the diagnostic ability of the latter (5). Therefore, CE may select the patients who should undergo DBE, either for diagnostic or therapeutic purposes, and it may determine the ideal insertion route for DBE (1).

This is the first study to assess and correlate the diagnostic performances of CE and ${ }^{99 m}$ Tc RBC scintigraphy. In fact, this study aimed to evaluate the degree of concordance between scintigraphy results and the results obtained from a high diagnostic yield method with direct visualization of the small bowel. The distribution of findings with hemorrhagic potential was similar in patients with negative and positive scintigraphy, and the concordance of the results of both methods was less than that expected by chance. This observation is in line with findings reported by other authors. Speir et al. reported an overall concordance of $29 \%$ between nuclear scan and angiography, and 
reported a weaker correlation with increasing intervals between the tests (8). Radiolabeled RBC scintigraphy does not accurately pinpoint the location of hemorrhagic foci. The blush is most frequently referred as occurring in a certain abdominal region, and from this information a broad prediction of the bleeding source is determined. No concordance was found between the presumed location of the bleeding on scintigraphy and the location of lesions with bleeding potential on CE. These data are in accordance with those obtained by Feuerstein et al., in which ${ }^{99 \mathrm{~m} T c}$ RBC scintigraphy performed worse than CT angiography for the precise localization of significant bleeding lesions (12). Finally, the values of hemoglobin, which is a surrogate marker for severity of bleeding, did not differ between patients with and without positive scans $(p=0.058)$. This may reflect the limitations of ${ }^{99 m} \mathrm{Tc}$ RBC scintigraphy to predict the severity of hemorrhage. Furthermore, Duraiswamy and colleagues did not find any differences in hematocrit values between those with positive and negative scintigraphy (9). In contrast, patients with P2 (high bleeding potential) lesions on CE had statistically significant lower levels of hemoglobin at presentation than those with PO (no bleeding potential) lesions. Moreover, the existence of lesions with a high bleeding potential moderately correlated with lower levels of hemoglobin. Similar results were obtained by other groups (18).

This study has merit as it is the first to confront the diagnostic performances of scintigraphy, a widely used test, and CE, the method with the highest diagnostic yield. All examinations were performed in the Gastroenterology and Nuclear Medicine Departments of the same hospital by highly trained medical professionals. However, this study has several limitations. First, it is a single-center retrospective study. Second, the number of patients included was relatively small, which may affect the generalizability of our results. Third, most of these patients were seen for irondeficient anemia in multiple medical specialties. Therefore, medical practices regarding the request of $\mathrm{CE}$ or scintigraphy may have varied markedly. Fourth, the interval between CE and scintigraphy was large. This may have contributed to discordant findings between CE and scintigraphy. The effect of wider intervals may be particularly important for intermittent or short-lived bleeds. Moreover, therapeutic strategies implemented during this interval were not accounted for. Therefore, although a sub- 
analysis demonstrated similar results between those with intervals over two months and those not exceeding two months, the potential influence of this interval on the final results cannot be ignored. Finally, information regarding symptoms, current medications, and other risk factors for OGIB (e.g., NSAIDs) was not systematically reported in medical files, thus hindering an assessment of these variables.

In conclusion, this study reinforces the existing evidence questioning the role of scintigraphy for the workup of patients with OGIB. In fact, no concordance was observed between the scintigraphy results and those of a high diagnostic yield technique such as CE. The latter has proven to be an efficient diagnostic tool and the most cost-effective approach to OGIB patients. On the other hand, the investigation, follow-up, and treatment of patients with OGIB is rarely determined by scintigraphy results, whereas CE influences subsequent follow-up for most patients. Therefore, the results presented in this study lead us to question the true clinical usefulness of ${ }^{99 \mathrm{~m} T c}$ RBC scintigraphy when caring for these patients. 


\section{REFERENCES}

1. Pennazio M, Spada C, Eliakim R, et al. Small-bowel capsule endoscopy and device-assisted enteroscopy for diagnosis and treatment of small-bowel disorders: European Society of Gastrointestinal Endoscopy (ESGE) Clinical Guideline. Endoscopy 2015;47(4):352-76. DOI: 10.1055/s-0034-1391855

2. Leung WK, Ho SS, Suen BY, et al. Capsule endoscopy or angiography in patients with acute overt obscure gastrointestinal bleeding: a prospective randomized study with long-term follow-up. Am J Gastroenterol 2012;107(9):1370-6. DOI: 10.1038/ajg.2012.212

3. Laine L, Sahota A, Shah A. Does capsule endoscopy improve outcomes in obscure gastrointestinal bleeding? Randomized trial versus dedicated small bowel radiography. Gastroenterology 2010;138(5):1673-80.e1; quiz e11-2. DOI: 10.1053/j.gastro.2010.01.047

4. Triester SL, Leighton JA, Leontiadis GI, et al. A meta-analysis of the yield of capsule endoscopy compared to other diagnostic modalities in patients with obscure gastrointestinal bleeding. Am J Gastroenterol 2005;100(11):2407-18. DOI: $10.1111 / j .1572-0241.2005 .00274 . x$

5. Teshima CW, Kuipers EJ, van Zanten SV, et al. Double balloon enteroscopy and capsule endoscopy for obscure gastrointestinal bleeding: an updated metaanalysis. J Gastroenterol Hepatol 2011;26(5):796-801. DOI: $10.1111 / j .1440-1746.2010 .06530 . x$

6. Shim K-N, Moon JS, Chang DK, et al. Guideline for Capsule Endoscopy: Obscure Gastrointestinal Bleeding. Clin Endosc 2013;46(1):45-53. DOI: 10.5946/ce.2013.46.1.45

7. Farhat R, Kim DT, French TD, et al. A Novel Technique to Measure the Intensity of Abnormality on GI Bleeding Scans: Development, Initial Implementation, and Correlation With Conventional Angiography. Clin Nucl Med 2018;43(2):82-6. DOI: $10.1097 /$ rlu. 0000000000001920

8. Speir EJ, Newsome JM, Bercu ZL, et al. Correlation of CT Angiography and (99m)Technetium-Labeled Red Blood Cell Scintigraphy to Catheter Angiography 
for Lower Gastrointestinal Bleeding: A Single-Institution Experience. J Vasc Interv Radiol 2019;30(11):1725-32.e7. DOI: 10.1016/j.jvir.2019.04.019

9. Duraiswamy S, Schmulewitz N, Rockey DC. The role of $99 \mathrm{~m}$ Tc-RBC scintigraphy in lower gastrointestinal hemorrhage. J Investig Med 2016;64(4):854-60. DOI: 10.1136/jim-2016-000077

10. Rondonotti E, Spada C, Adler S, et al. Small-bowel capsule endoscopy and device-assisted enteroscopy for diagnosis and treatment of small-bowel disorders: European Society of Gastrointestinal Endoscopy (ESGE) Technical Review. Endoscopy 2018;50(4):423-46. DOI: 10.1055/a-0576-0566

11. Saurin JC, Delvaux M, Gaudin JL, et al. Diagnostic value of endoscopic capsule in patients with obscure digestive bleeding: blinded comparison with video pushenteroscopy. Endoscopy 2003;35(7):576-84. DOI: 10.1055/s-2003-40244

12. Feuerstein JD, Ketwaroo G, Tewani SK, et al. Localizing Acute Lower Gastrointestinal Hemorrhage: CT Angiography Versus Tagged RBC Scintigraphy. AJR Am J Roentgenol 2016;207(3):578-84. DOI: 10.2214/ajr.15.15714

13. Fisher L, Lee Krinsky $M$, Anderson $M A$, et al. The role of endoscopy in the management of obscure GI bleeding. Gastrointest Endosc 2010;72(3):471-9. DOI: 10.1016/j.gie.2010.04.032

14. Gurajala RK, Fayazzadeh E, Nasr E, et al. Independent usefulness of flow phase (99)mTc-red blood cell scintigraphy in predicting the results of angiography in acute gastrointestinal bleeding. Br J Radiol 2019;92(1094):20180336. DOI: 10.1259/bjr.20180336

15. Liao Z, Gao R, Xu C, et al. Indications and detection, completion, and retention rates of small-bowel capsule endoscopy: a systematic review. Gastrointest Endosc 2010;71(2):280-6. DOI: 10.1016/j.gie.2009.09.031

16. Marmo R, Rotondano G, Rondonotti E, et al. Capsule enteroscopy vs. other diagnostic procedures in diagnosing obscure gastrointestinal bleeding: a costeffectiveness study. Eur J Gastroenterol Hepatol 2007;19(7):535-42. DOI: 10.1097/MEG.0b013e32812144dd

17. Gurudu SR, Bruining DH, Acosta RD, et al. The role of endoscopy in the management of suspected small-bowel bleeding. Gastrointest Endosc 
2017;85(1):22-31. DOI: 10.1016/j.gie.2016.06.013

18. Wetwittayakhlang $\mathrm{P}$, Wonglhow J, Netinatsunton $\mathrm{N}$, et al. Re-bleeding and its predictors after capsule endoscopy in patients with obscure gastrointestinal bleeding in long-term follow-up. BMC Gastroenterol 2019;19(1):216. DOI: $10.1186 / \mathrm{s} 12876-019-1137-3$

19. Otani K, Watanabe T, Shimada S, et al. Clinical Utility of Capsule Endoscopy and Double-Balloon Enteroscopy in the Management of Obscure Gastrointestinal Bleeding. Digestion 2018;97(1):52-8. DOI: 10.1159/000484218

20. Ribeiro I, Pinho R, Rodrigues A, et al. Obscure gastrointestinal bleeding: Which factors are associated with positive capsule endoscopy findings? Rev Esp Enferm Dig 2015;107(6):334-9.

21. Shahidi NC, Ou G, Svarta S, et al. Factors associated with positive findings from capsule endoscopy in patients with obscure gastrointestinal bleeding. Clin Gastroenterol Hepatol 2012;10(12):1381-5. DOI: 10.1016/j.cgh.2012.08.035

22. Shimada S, Watanabe T, Nadatani $Y$, et al. Clinical factors associated with positive capsule endoscopy findings in patients with obscure gastrointestinal bleeding: a single-center study. Scand J Gastroenterol 2017;52(11):1219-23. DOI: $10.1080 / 00365521.2017 .1349174$

23. Saperas E, Dot J, Videla S, et al. Capsule endoscopy versus computed tomographic or standard angiography for the diagnosis of obscure gastrointestinal bleeding. Am J Gastroenterol 2007;102(4):731-7. DOI: 10.1111/j.1572-0241.2007.01058.x 
Table 1. Baseline characteristics of patients

\begin{tabular}{|c|c|}
\hline Patient characteristics & $n(\%)$ or median (IQR) \\
\hline Female, n (\%) & $53(62 \%)$ \\
\hline Age, median (IQR) & $63(51-74)$ \\
\hline \multicolumn{2}{|l|}{ Indication for study } \\
\hline Occult OGIB, n (\%) & $73(86 \%)$ \\
\hline Overt OGIB, n (\%) & $12(14 \%)$ \\
\hline Hemoglobin (g/dL), median (IQR) & $10.2(8.9-10.9)$ \\
\hline \multicolumn{2}{|l|}{ Positive scintigraphy, n (\%) } \\
\hline \multicolumn{2}{|l|}{ Capsule enteroscopy } \\
\hline PO, n (\%) & $37(44 \%)$ \\
\hline $\mathrm{P} 1, \mathrm{n}(\%)$ & $11(13 \%)$ \\
\hline $\mathrm{P} 2, \mathrm{n}(\%)$ & $37(44 \%)$ \\
\hline \multicolumn{2}{|l|}{ Lesions ( $n=49$ ) } \\
\hline Angiectasia, n (\%) & $18(37 \%)$ \\
\hline Erosions, n (\%) & $10(20 \%)$ \\
\hline Ulcers, n (\%) & $6(12 \%)$ \\
\hline Petechiae, n (\%) & $6(12 \%)$ \\
\hline Lymphangiectasia, n (\%) & $5(10 \%)$ \\
\hline Other, n (\%) & $4(8 \%)$ \\
\hline $\begin{array}{l}\text { Time between CE and scintigraph } \\
\text { (IQR) }\end{array}$ & $4(1.5-7.0)$ \\
\hline
\end{tabular}

IQR: interquartile range; OGIB: obscure gastrointestinal bleeding; CE: capsule enteroscopy. 
Table 2. Distribution of capsule enteroscopy findings according to scintigraphy results

\begin{tabular}{l|ll}
\hline \multicolumn{2}{c}{ Positive scintigraphy } & Negative scintigraphy \\
\hline P0, n (\%) & $32(46 \%)$ & $5(33 \%)$ \\
P1, n (\%) & $8(11 \%)$ & $3(20 \%)$ \\
P2, n (\%) & $30(43 \%)$ & $7(47 \%)$ \\
\hline
\end{tabular}


Table 3. Distribution of capsule enteroscopy and scintigraphy findings according to the intervals between both diagnostic modalities

\begin{tabular}{lllll}
\hline & \multicolumn{3}{l}{ Interval between CE and scintigraphy } \\
\cline { 2 - 5 } & \multicolumn{2}{l}{ 2 months } & \multicolumn{2}{l}{ 2 months } \\
\cline { 2 - 5 } & Negative & Positive & Negative & Positive \\
\hline scintigraphy & scintigraphy & scintigraphy & scintigraphy \\
\cline { 2 - 5 } P0, n (\%) & $4(40 \%)$ & $24(50 \%)$ & $1(20 \%)$ & $8(36 \%)$ \\
P1, n (\%) & $2(20 \%)$ & $5(10 \%)$ & $1(20 \%)$ & $3(14 \%)$ \\
P2, n (\%) & $4(40 \%)$ & $19(40 \%)$ & $3(60 \%)$ & $11(50 \%)$ \\
\hline
\end{tabular}


Table 4. Median hemoglobin in scintigraphy and capsule enteroscopy

\begin{tabular}{lll}
\hline & Median hemoglobin & $p$ \\
\hline Scintigraphy & $10.8(9.6-11.8)$ & 0.058 \\
Negative & $10.1(8.8-10.8)$ & $0.002^{*}$ \\
Positive & $10.6(9.7-11.5)$ & \\
Capsule enteroscopy & $10.3(9.3-11.5)$ \\
P0 & $9.3(8.1-10.5)$ \\
P1 &
\end{tabular}

IQR: interquartile range. ${ }^{*} p<0.05$ 


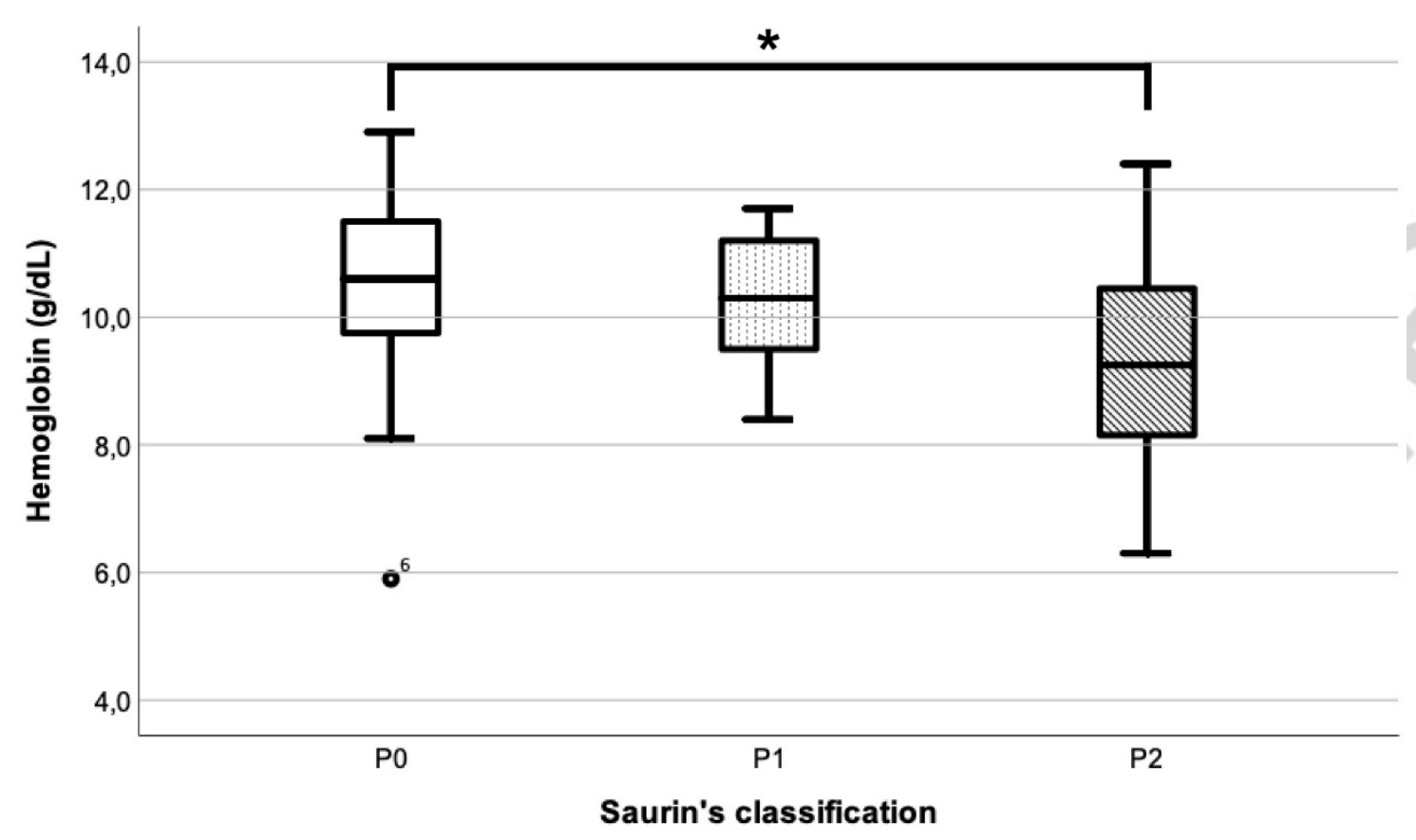

Fig. 1. Boxplot demonstrating the median values of hemoglobin across capsule enteroscopy findings (according to Saurin's classification). Median hemoglobin levels for high bleeding potential lesions (P2) were significantly lower than for lesions without bleeding potential (PO). ${ }^{*} p<0.05$. 